\title{
Selection for behavior, intermale confrontations, and corticosterone and testosterone levels in the blood of norway rats
}

\author{
R. Gulevich ${ }^{1 *}$, R. Kozhemyakina ${ }^{1}$, M. Konoshenko², S. Shikhevich ${ }^{1}$ \\ ${ }^{1}$ Institute of Cytology and Genetics SB RAS, Novosibirsk, Russia \\ ${ }^{2}$ Institute of Chemical Biology and Fundamental Medicine, Novosibirsk, Russia \\ *e-mail: gulevich@bionet.nsc.ru
}

Key words: aggressiveness, tameness, selection, rats, confrontations, neutral area

Motivation and Aim: Aggression is believed to be promoted by various factors: hereditary, environmental, developmental, hormonal, and neurotransmitter-related. In some scientists' view, testosterone contributes to aggression manifestation, and this contribution increases with an imbalance of other factors, such as low cortisol levels. This work concerns the effect of selection for attitude towards humans on the dynamics of blood testosterone, corticosterone after the test for intermale aggression in an unfamiliar cage and on the parameters of agonistic contacts under the same conditions in tame, aggressive, and unselected rats.

Methods and Algorithms: Experiments were conducted with two-month-old rat males (Rattus norvegicus) of the $79^{\text {th }}$ generation of selection for absence and enhancement of the aggressive-fearful response to humans. Intermale aggression was tested in an unfamiliar transparent plastic cage, The cage was partitioned into two compartments. Male rats were placed by ones into the compartments, and the partition was removed. Eighteen tests were done: six between unselected animals, six between aggressive, and six between tame. Behavior parameters were recorded for 5 min: attack latency; number and durations of attacks, chasings, kickings, upright postures, pinning, wrestling, aggressive grooming, lateral threats. Blood was sampled from the tail tip immediately and within 30,60, and $120 \mathrm{~min}$ after the intermale aggression test.

Results: The attack latency is longer in tame males than in aggressive or unselected ones, whereas the number and duration of aggressive behaviors are less. Confrontations between unselected males in a neutral area lasted for longer than between aggressive ones. After confrontations, the level of corticosterone increases, being higher in unselected animals than in aggressive or tame within two hours after the test, as well as in the intact state. Although blood testosterone levels show no significant differences among the three groups prior to the test, tame rats are inferior in this index to unselected and aggressive animals immediately after the test, and only to aggressive animals within 30 min after a confrontation.

Conclusion: Our data on the parameters of behavior in a neutral area indicate that aggressiveness in tame males is reduced in comparison to not only aggressive animals, but also to unselected ones. The fact that confrontations between unselected males are longer than between aggressive may be determined by the selection criterion itself, because the original response to a glove was related to the latency time rather than aggression duration. Post-confrontation corticosterone in unselected rats was higher than in others and post-confrontation testosterone in tame rats was lower than in other ones. Acknowledgements: Supported by the State Budgeted Project 0324-2018-0016. 